\title{
3D printing of patient-specific neck splints for the treatment of post-burn neck contractures
}

\author{
Dafydd O. Visscher ${ }^{1,2^{*}}$ D, Sjoerd te Slaa ${ }^{2,4}$, Mariëlle E. Jaspers ${ }^{1,5}$, Marloes van de Hulsbeek ${ }^{6}$, Jorien Borst ${ }^{6}$, \\ Jan Wolff', ${ }^{2,3}$ Tymour Forouzanfar ${ }^{2,3}$ and Paul P. van Zuijlen ${ }^{1,5}$
}

Keywords: Neck burns, Contracture, 3D printing, Neck splint, Clinical, Optical 3D scanning

\section{Dear Editor,}

Burn scar contracture is a common problem in healing burn wounds of the neck. It can cause both pain and dysfunction if not treated adequately [1]. The treatment of such wounds often involves a combination of surgery and splinting therapy [2]. A variety of splints, including the thermoplastic static neck splint [3], the Watusi collar [4], manually fabricated splints, and pre-fabricated splints such as the Philadelphia collar have been used for the management of scar contractures. However, each type of splint has its own advantages and disadvantages, and none of these splints seem to reduce the need for skin reconstruction nor delays the time until surgical reconstruction [5].

Medical applications for optical three-dimensional (3D) scanning and 3D printing are evolving rapidly [6], and both technologies could revolutionize the field of burn and wound care. More specifically, optical 3D scanners in combination with 3D printing technologies can be used to manufacture patient-specific devices such as splints for the treatment of post-burn neck contractures. The combination of these technologies could increase product customization, production speed, and cost-effectiveness of splint development. 3D printing is already being used for surgical planning, education, and implant customization [7]. Furthermore, facial masks have already been manufactured for burn patients using optical 3D scanning and 3D printing technologies $[8,9]$.

\footnotetext{
* Correspondence: d.visscher@vumc.nl

'Department of Plastic, Reconstructive and Hand Surgery, Amsterdam Movement Sciences, VU University Medical Center, de Boelelaan 1117, 1081 HV Amsterdam, the Netherlands

23D InnovationLab, VU University Medical Center, Amsterdam, the Netherlands

Full list of author information is available at the end of the article
}

The aim of this study was to determine whether clinical use of these technologies for the production of patientspecific neck splints is feasible in a group of burn patients.

A retrospective study was performed with six patients who had been treated for burns and burn-related neck contractures at the Red Cross Hospital Burn Center in Beverwijk, the Netherlands. Following admission, all patients with neck burns were scanned using an optical 3D scanner (Artec Spider ${ }^{\text {tw }}$; Artec Group, Moscow, Russia). Following scanning, all patients received a 3D-printed neck splint consisting of silicone and medical-grade nylon (Fig. 1a-c) instead of a standard neck splint (which in our burn center is a manually fabricated neck splint). In order to determine patient satisfaction, a telephonic questionnaire was administered to all patients (Table 1). The study was approved by the regional Medical Ethics Committee of Noord-Holland, the Netherlands (M016-004). In light of the Declaration of Helsinki, all patients gave oral informed consent to start the intervention. Additional written informed consent was obtained from patients whose photographs were used for publication.

One engineer with in-depth knowledge in medical 3D scanning performed all optical 3D scans, including postprocessing. Optical 3D scanning took $30 \mathrm{~min}$, including setup (Fig. 1d). Computer-aided design was completed in approximately $4 \mathrm{~h}$. 3D printing of the splints took approximately 4 days because not all the parts could not be printed in-house. Therefore, the total production time for one 3D-printed neck splint was 5 days. The thickness of the final silicone splints and overlying nylon honeycomb scaffolds were 5 and $3 \mathrm{~mm}$ respectively.

The median follow-up after the initiation of the 3Dprinted splints was 7 months (interquartile range (IQR): 

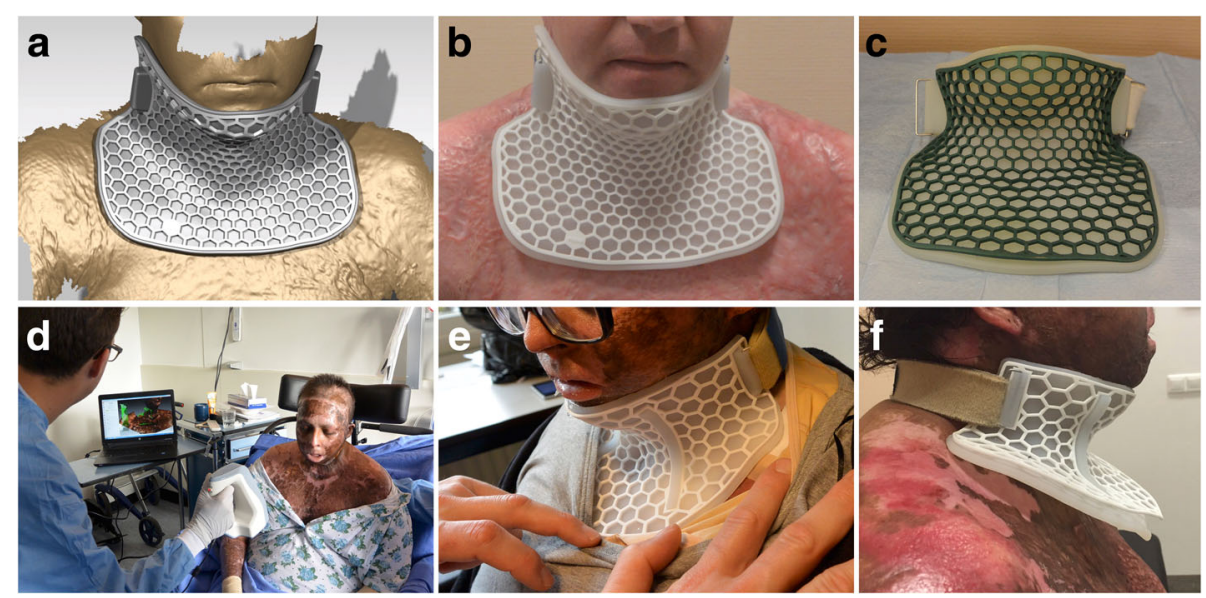

Fig. 1 Design and three-dimensional (3D) printing of patient-specific splints for neck burns. a Computer-aided design of neck splint. b 3D-printed neck splint on the same patient. c 3D-printed neck splint in color (for patient 5). d Contactless optical 3D scanning process. e 3D-printed neck splint on the same patient. $\mathbf{f}$ three months after wearing neck splint (note anatomical change)

4-16.8) (Table 1). The comfort levels of the 3D-printed splints were satisfactory (6.8, IQR: 5.1-8.1). Patients wore their splints for long periods during the day $(7.5 \mathrm{~h}, \mathrm{IQR}$ : 4.3-14h), but only two out of six patients wore their splints at night (during sleep). The splints were graded very well overall with a score of 8 out of a maximum 10 points (IQR: 5.9-8.1). Patients suggested to improve the splint straps since they caused itching and occasionally neck pain (Table 1, Fig. 1e-f). All splints fitted well and no major complications were observed.

In this study, we show that patient-specific neck splints can be fabricated using optical 3D scanning and $3 \mathrm{D}$ printing technology. With current advances in optical 3D scanning, computer-aided design, and 3D printing, patient-specific neck splints can be manufactured precisely and contactless; patients could be scanned at any given time, even in the operating room. The fabrication workflow took approximately 5 days to complete.
Although this is faster than the manually fabricated splints ( 7 days) at our burn center, many other splints can be fabricated much faster (e.g., Watusi collar and Philadelphia collar). In the near future, mobile phones with scanning software might be accurate enough to perform a scan of the neck area, which subsequently accelerates the production process [10].

The production costs for patient-specific neck splints are higher than for currently available splints. Therefore, clinical application of 3D-printed splints is, at this point, not yet cost-effective. If the costs could be reduced by using cheaper optical 3D scanners and free open source CAD software, patient-specific splints might become available for widespread clinical application in the near future.

One big advantage of using CAD software for modeling of splints is the ease of changing specific parameters during wound healing. Although some other splints can

Table 1 Patient demographics and response to questionnaire following wearing of three-dimensional (3D)-printed neck splint

\begin{tabular}{|c|c|c|c|c|c|c|c|c|c|c|}
\hline \multicolumn{6}{|c|}{ Patient demographics } & \multicolumn{5}{|c|}{ Patient response } \\
\hline Patient & Age (years) & TBSAB (\%) & $\begin{array}{l}\text { Initiation of } \\
\text { splint (days) }\end{array}$ & $\begin{array}{l}\text { No. of splints } \\
\text { received }\end{array}$ & $\begin{array}{l}\text { Follow-up } \\
\text { (months) }\end{array}$ & $\begin{array}{l}\text { Comfortability } \\
(1-10)\end{array}$ & $\begin{array}{l}\text { Daytime wear } \\
\text { (hours) }\end{array}$ & $\begin{array}{l}\text { Nighttime } \\
\text { wear }\end{array}$ & Suggestions & $\begin{array}{l}\text { Overall } \\
\text { grade }\end{array}$ \\
\hline $1(\mathrm{M})$ & 42 & 56.5 & 29 & 3 & 19 & 8.5 & 7 & No & Strap & 8.5 \\
\hline $2(F)$ & 43 & 60 & 13 & 2 & 16 & 7 & 14 & No & - & 8 \\
\hline $3(\mathrm{M})$ & 72 & 18 & 46 & 1 & 1 & 8 & 5 & No & Strap & 8 \\
\hline $4(\mathrm{~F})$ & 25 & 18 & 31 & 1 & 7 & 1 & 2 & No & - & 1 \\
\hline $5(M)$ & 9 & 31 & 14 & 4 & 7 & 6.5 & 8 & Yes & Strap & 7.5 \\
\hline $6(M)$ & 42 & 44.6 & 4 & 2 & 5 & 6.5 & 14 & Yes & Strap & 8 \\
\hline $\begin{array}{l}\text { Median } \\
\text { (Q1-Q3) }\end{array}$ & $42(21-50.3)$ & $37.8(18-57.4)$ & $21.5(10.8-34.8)$ & $2(1-3.3)$ & $7(4-16.8)$ & $6.8(5.1-8.1)$ & $7.5(4.3-14)$ & - & - & $8(5.9-8.1)$ \\
\hline
\end{tabular}

Daytime wear (hours) refers to the number of hours the patient wore the splint during the day. Nighttime wear refers to whether the patient wore the splint while sleeping.

Q1: 25th percentile, Q3: 75th percentile

$M$ male, $F$ female, TBSAB total body surface area burned 
also be adjusted after anatomical change (e.g., thermoplastic splints), these always require patient contact to fit perfectly. In our study, most patients required additional splints due to anatomical changes during wound healing (Fig. 1f). This problem could be solved rapidly by designing a splint to fit the new anatomical situation. This iterative process optimizes patient treatment and could eventually contribute to the treatment of burns in remote areas. The authors believe that in the near future, parametric modeling may contribute to the development of bioinspired splints with pressure distribution and even local drug release.

\section{Acknowledgements}

The authors thank Niels Liberton and Frank Verver for their help with software processing and optical 3D scanning

\section{Funding}

This study was financially supported by a grant from the Dutch Burn Foundation (15.107). The funding did not include roles in study design or writing.

\section{Availability of data and materials}

Additional data, including the methods used to fabricate the product in this study is available upon reasonable request.

\section{Authors' contributions}

DV collected and interpreted the data and wrote the manuscript. SS collected the data, helped with the data interpretation, converted the data to $3 \mathrm{D}$ printing form, and revised the manuscript. $\mathrm{MJ}$ collected data and helped in writing the manuscript. MvdH and JB helped with patient inclusion and helped with the data collection and revised the manuscript. JW and TF provided software and hardware to conduct the study, helped writing the manuscript, and critically revised the manuscript. PVZ helped with patient inclusion and to define the research question, critically revised the manuscript, and approved the final manuscript. All authors read and approved the final manuscript.

\section{Ethics approval and consent to participate}

The study was approved by the regional Medical Ethics Committee of Noord-Holland, the Netherlands (M016-004). In light of the Declaration of Helsinki, all patients gave oral informed consent to start the intervention. Additional written informed consent was obtained from patients whose photographs were used for publication.

\section{Consent for publication}

Consent for publication was obtained from patients whose photographs have been used in this study.

\section{Competing interests}

The authors declare that they have no competing interests.

\section{Author details}

${ }^{1}$ Department of Plastic, Reconstructive and Hand Surgery, Amsterdam Movement Sciences, VU University Medical Center, de Boelelaan 1117. 1081HV Amsterdam, the Netherlands. ${ }^{2} 3 \mathrm{D}$ InnovationLab, VU University Medical Center, Amsterdam, the Netherlands. ${ }^{3}$ Department of Oral and Maxillofacial Surgery/Oral Pathology, VU University Medical Center, Amsterdam, the Netherlands. ${ }^{4}$ Department of Medical Technology, VU University Medical Center, Amsterdam, the Netherlands. ${ }^{5}$ Department of Plastic, Reconstructive and Hand Surgery/Burn Center, Red Cross Hospital, Beverwijk, the Netherlands. ${ }^{6}$ Department of Occupational Therapy, Red Cross Hospital, Beverwijk, the Netherlands.
Received: 26 December 2017 Accepted: 25 April 2018

Published online: 08 June 2018

\section{References}

1. Yang J-Y, Tsai F-C, Chana JS, Chuang S-S, Chang S-Y, Huang W-C. Use of free thin anterolateral thigh flaps combined with cervicoplasty for reconstruction of postburn anterior cervical contractures. Plast Reconstr Surg. 2002;110:39-46.

2. Richard R, Ward RS. Splinting strategies and controversies. J Burn Care Rehabil. 2005;26:392-6.

3. Fowler D, Pegg SP. Evaluation of aquaplast splinting thermoplastic - use in a burns unit. Burns Incl Therm Inj. 1983;9:284-7.

4. Hurlin Foley K, Doyle B, Paradise P, Parry I, Palmieri T, Greenhalgh DG. Use of an improved Watusi collar to manage pediatric neck burn contractures. J Burn Care Rehabil. 2002;23:221-6.

5. Sharp PA, Dougherty ME, Kagan RJ. The effect of positioning devices and pressure therapy on outcome after full-thickness burns of the neck. J Burn Care Res Off Publ Am Burn Assoc. 2007;28:451-9.

6. Choonara YE, du Toit LC, Kumar P, Kondiah PPD, Pillay V. 3D-printing and the effect on medical costs: a new era? Expert Rev Pharmacoecon Outcomes Res. 2016;16:23-32.

7. Hoang D, Perrault D, Stevanovic M, Ghiassi A. Surgical applications of threedimensional printing: a review of the current literature \& how to get started. Ann Transl Med. 2016:4:456.

8. Wei Y, Li-Tsang CWP, Liu J, Xie L, Yue S. 3D-printed transparent facemasks in the treatment of facial hypertrophic scars of young children with burns. Burns J Int Soc Burn Inj. 2017;43:e19-26.

9. Pilley MJ, Hitchens C, Rose G, Alexander S, Wimpenny DI. The use of noncontact structured light scanning in burns pressure splint construction. Burns J Int Soc Burn Inj. 2011;37:1168-73.

10. Ondrúška $P$, Kohli $P$, Izadi S. Mobilefusion: real-time volumetric surface reconstruction and dense tracking on mobile phones. IEEE Trans Vis Comput Graph. 2015:21:1251-8. 